\title{
Necrosis retiniana aguda por virus varicela zoster en un paciente inmunocompetente con seguimiento de la respuesta terapéutica mediante reacción de polimerasa en cadena
}

\author{
Acute retinal necrosis by varicella zoster virus in an immunocompetent patient with \\ therapeutic follow up by PCR
}

\author{
Alberto Fica 1,5, Diana Aceituno2, Alba Montecinos 3,6, Maritza Navarrete4, Marcelo Ojeda33, Eduardo Carrasco ${ }^{3,7}$ e Israel Giacaman ${ }^{4}$
}

\begin{abstract}
'Subdepartamento de Medicina, Hospital Base de Valdivia. Valdivia, Chile.
${ }^{2}$ Unidad de Hematología Intensiva, Hospital del Salvador. Santiago, Chile.

${ }^{3}$ Servicio de Oftalmología, Hospital Base de Valdivia. Valdivia, Chile.

${ }^{4}$ Laboratorio de Biología Molecular, Hospital Base de Valdivia. Valdivia, Chile.

Instituto de Medicina, Universidad Austral de Chile. Valdivia, Chile.

שUnidad de Oftalmología, Instituto de Especialidades, Facultad de Medicina, Sede Valdivia, Universidad Austral de Chile. Valdivia, Chile.

${ }^{7}$ Escuela Tecnología Médica, Sede Puerto Montt, Universidad Austral de Chile. Valdivia, Chile.
\end{abstract}

Fuente financiamiento: ninguna.

Conflicto de interés: ninguno.

Recibido: 17 de enero de 2021 / Aceptado: 26 de abril de 2021

\section{Resumen}

Presentamos el caso de un varón de 63 años, inmunocompetente, con una necrosis retinal aguda (NRA) unilateral. Consultó por visión borrosa, dolor ocular, fotofobia y cefalea. Se confirmó una papilitis y coriorretinitis periférica asociada a vasculitis e isquemia retinal periférica. El estudio molecular por RPC de humor acuoso detectó la presencia de virus varicela zoster. El paciente fue tratado con terapia combinada con corticoesteroides orales, aciclovir oral/intravenoso, ganciclovir intravítreo semanal y luego valaciclovir oral por tres meses. Se demostró una disminución progresiva de la carga viral en el humor acuoso durante el tratamiento. El seguimiento mostró una mejoría del cuadro inflamatorio y una leve recuperación de la agudeza visual, sin embargo, finalmente presentó un desprendimiento de retina con pérdida casi total de la visión unilateral. La NRA es una complicación infrecuente provocada por algunos virus herpes con mal pronóstico visual, desenlace que puede ser mejorado con un diagnóstico y tratamiento precoz con antivirales. El tratamiento prolongado permite evitar la recaída y el compromiso contralateral.

Palabras claves: síndrome necrosis retinal aguda; infección por virus varicela zoster; reacción en cadena de la polimerasa a tiempo real; aciclovir; valaciclovir; desprendimiento de retina.

\section{Abstract}

We present the case of a 63-year-old immunocompetent man with unilateral acute retinal necrosis (ARN). He consulted for blurred vision, eye pain, photophobia, and headache. Papillitis and peripheral chorioretinitis associated with vasculitis and peripheral retinal ischemia was confirmed. PCR from aqueous humor sample detected varicella zoster virus. The patient was treated with a combined therapy of oral corticosteroids, oral / intravenous acyclovir along with weekly intravitreous ganciclovir doses followed by oral valaciclovir for three months. A progressive decrease in viral load in aqueous humor was demonstrated during treatment. Follow-up showed improvement in the inflammatory condition and a slight recovery of visual acuity, however, finally he presented a retinal detachment with total loss of one-sided vision. ARN is an uncommon complication caused by some herpesviruses with a poor visual prognosis, an outcome that can be improved with early diagnosis and treatment using appropriate antivirals. Prolonged treatment reduces relapse frequency and fellow eye compromise.

Keywords: retinal necrosis syndrome, acute; varicella zoster virus infection; real time polymerase chain reaction; acyclovir; valacyclovir; retinal detachment. 


\section{Introducción}

L

a necrosis retinal aguda (NRA) es una manifestación grave de la infección por virus herpes simplex (VHS), citomegalovirus (CMV) o varicela zoster (VVZ). Ocurre generalmente por una reactivación viral y se expresa con visión borrosa o pérdida de ésta, dolor a la movilización ocular y ojo rojo $^{1-3}$. Los síntomas se explican por uno o más focos progresivos de necrosis retinal periférica, diseminación circunferencial, vasculopatía oclusiva isquémica e inflamación en la cámara anterior y el vítreo ${ }^{4}$. Las consecuencias inmediatas o mediatas son la pérdida de la visión, desprendimiento de retina (DR) o el compromiso contralateral ${ }^{1,4,5}$.

Cada caso de NRA se confronta al problema diagnóstico para aclarar su etiología específica, al tratamiento antiviral y su duración y al pronóstico visual del paciente. Comunicamos el caso de una NRA por VVZ en el cual se hizo un seguimiento molecular y oftalmológico detallado.

\section{Caso clínico}

Varón de 63 años, con antecedentes de hipertensión arterial y dislipidemia en tratamiento con ácido acetilsalícilico, atorvastatina y enalapril. Consultó por visión borrosa del ojo izquierdo de cinco días de evolución asociado a dolor ocular, fotofobia y cefalea hemicraneal izquierda. No tenía antecedentes de trauma, mordedura o rasguño por animales, enfermedades de transmisión sexual o autoinmunes, o tuberculosis. El cuadro se presentó sin compromiso del estado general, tos, fiebre, adenopatías, artralgias o lesiones cutáneas o mucosas. Al examen oftalmológico se detectó una agudeza visual (AV) de 0,8 en el ojo derecho (OD) y 0,3 en el ojo izquierdo (OI), con una presión intraocular aumentada en el OI (22 mmHg). La exploración del OD no demostró hallazgos patológicos (Figura 1A). La biomicroscopía (BMC) del OI evidenció una hiperemia, córnea edematosa, precipitados retroqueráticos en grasa de carnero, fenómeno de Tyndall positivo, sin hipopion y cristalino claro. El fondo del OI reveló una papila hiperémica y la retina con múltiples infiltrados blancos de distribución difusa periférica de tamaño variable compatibles con coriorretinitis (Figura 1B). En una ecografía modo B del OI se pesquisó una imagen hiperecogénica compatible con un desprendimiento seroso de retina hacia la zona temporal inferior. La angiografía con fluoresceína (AGF) evidenció una vasculitis e isquemia retinal periférica, defecto pantalla en lesiones coriorretinales y fuga de fluoresceína a nivel papilar en tiempos tardíos (Figura 1C, 1D). Se planteó el diagnóstico de una panuveitis del OI iniciando tratamiento empírico con aciclovir oral (4 g al día), cotrimoxazol forte $(800 / 160 \mathrm{mg}) 5$ comprimidos al día en tres dosis y tratamiento tópico con corticoesteroides y midriáticos. En las pruebas de laboratorio inicial destacaron valores de hemoglobina y velocidad de eritrosedimentación normal. No se detectó eosinofilia y la serología para toxocariosis fue negativa.

Se efectuó un estudio para descartar diferentes causas de uveítis infecciosa o autoinmune. La radiografía de tórax fue normal, el factor reumatoideo negativo, valores de complemento, proteína $\mathrm{C}$ reactiva, recuento de inmunoglobulinas IgG, IgA, IgM, $\beta-2$ microglobulina y glicemia fueron normales. Asimismo, las serologías para VIH y sífilis fueron negativas y los resultados de la serologías IgG e IgM para CMV y toxoplasmosis indicaron una infección pasada (IgG positivo con IgM negativo). Tampoco había elementos clínicos de endocarditis infecciosa, ni lesiones mucocutáneas orogenitales que sugirieran un síndrome de Behçet, o compromiso auditivo, cutáneo, alopecia, vitiligo o encefalitis que plantearan un síndrome de Vogt-Koyanagi-Harada.

Con los resultados serológicos negativos para toxoplasmosis se decidió suspender la terapia con cotrimoxazol y se inició prednisona a dosis $1 \mathrm{mg} / \mathrm{kg} /$ día. La confluencia de las lesiones retinales fueron compatibles con una NRA por virus herpes: áreas de necrosis retiniana focal en la periferia con vasculopatía oclusiva e inflamación del humor vítreo y en la cámara anterior, por lo que se cambió la administración de aciclovir oral a vía intravenosa $(10 \mathrm{mg} / \mathrm{kg} / \mathrm{dosis}$ cada $8 \mathrm{~h})$ y se disminuyó la dosis de prednisona a $0,5 \mathrm{mg} / \mathrm{kg} / \mathrm{día}$. Además, se aplicó una primera dosis de ganciclovir intravítreo $(5 \mathrm{mg} / 0,1 \mathrm{ml})$ extrayendo una muestra de humor acuoso para estudio molecular con RPC, la que fue positiva para VVZ, sin que hubiera amplificación para CMV o VHS-1 o 2. En los siguientes días el paciente evolucionó con una mejoría subjetiva de la visión asociada a una resolución de los infiltrados subendoteliales, disminución del fenómeno Tyndall en cámara anterior, regresión del edema de papila, resolución de los infiltrados retinales del polo posterior y disminución progresiva de la necrosis retinal periférica asociado a cambios pigmentarios sin desprendimiento de retina, persistiendo los vasos retinales anormales. La angiografía evidenció zonas de isquemia periférica que se manejaron en forma expectante sin láser. Hasta el egreso a los 40 días, se aplicaron cuatro dosis intravítreas de ganciclovir semanal y se completaron 26 días de terapia con aciclovir intravenoso. Al alta se indicó terapia complementaria con valaciclovir $1,5 \mathrm{~g}$ cada $8 \mathrm{~h}$, por vía oral por tres meses, como terapia de mantención. Los estudios de RPC con las muestras obtenidas en cada inoculación de ganciclovir intravítrea revelaron un aumento progresivo del umbral de ciclo (27,9 a 32,3), (Figura 2) lo que sugiere una disminución progresiva de la carga viral. Se efectuaron tres controles ambulatorios durante la fase de mantención, demostrándose en ellos una reducción de la 
Figura 1. A: Retinografía del ojo derecho (OD) normal. B: Retinografía del ojo izquierdo (OI) tomada al ingreso. Muestra un vítreo turbio por inflamación que no permite ver detalles, lográndose visualizar papila discretamente hiperémica (flecha blanca). Se observa también vasos sanguíneos con contorno blanquecino sugerente de vasculitis (envainamiento vascular, flecha negra) e infiltrados retinales (flechas grises). C: Angiografía con fluoresceína del OI tomada al ingreso que evidencia isquemia retinal (ausencia de llenado vascular, flecha blanca) y exudación de fluoresceína a nivel papilar por inflamación (D, flecha blanca).

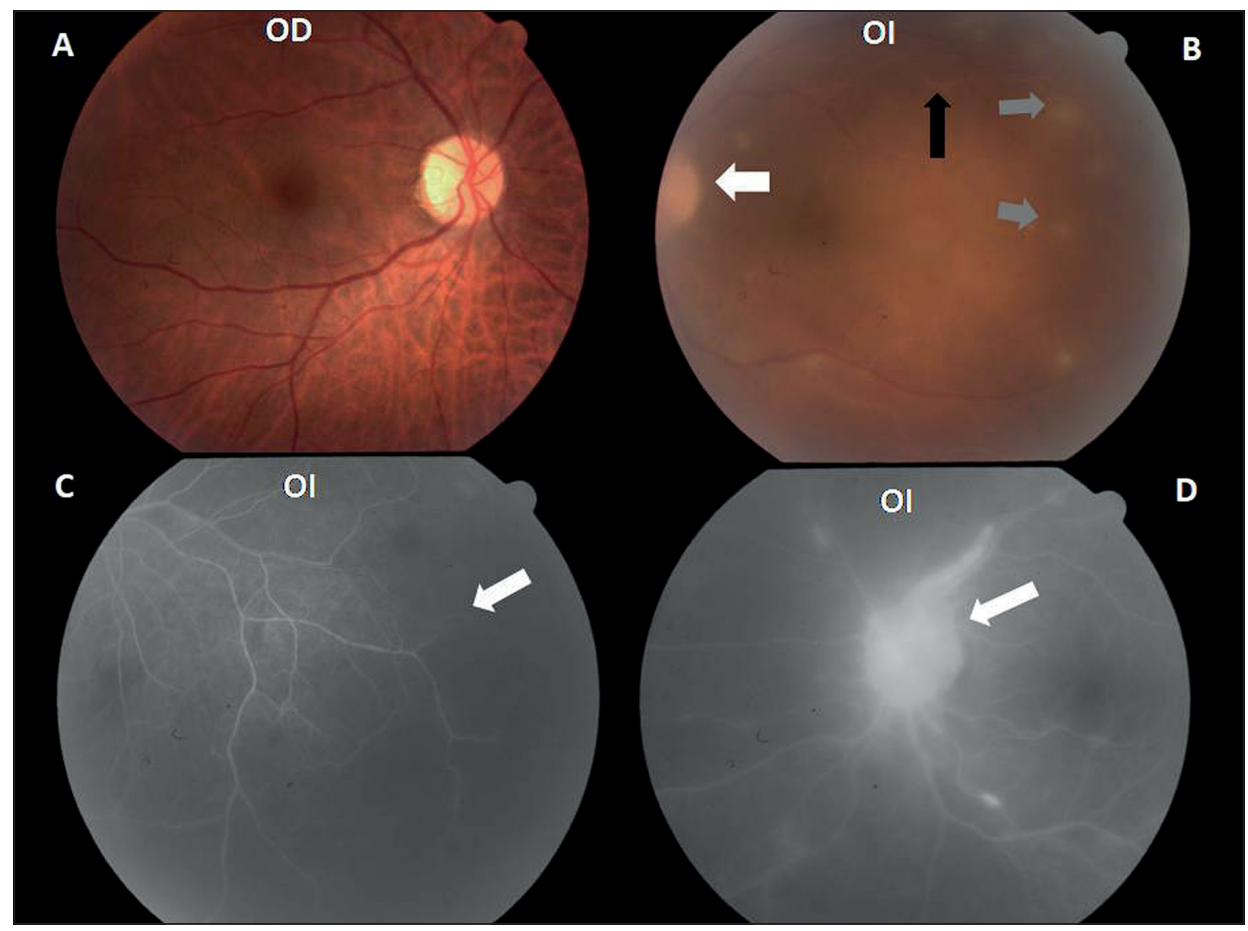

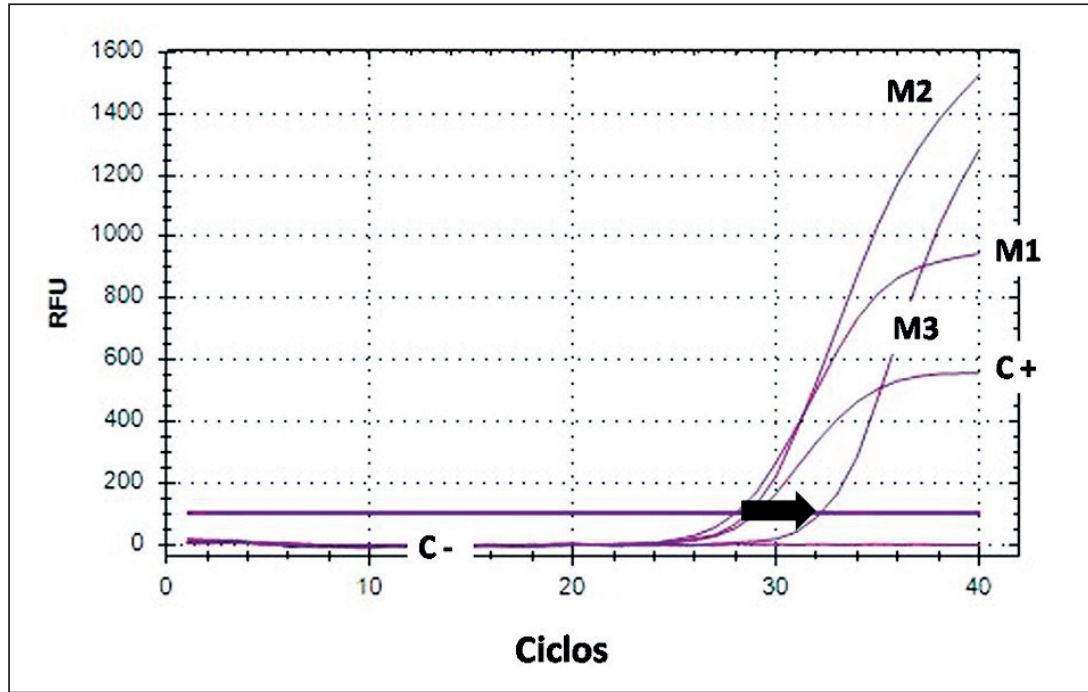

Figura 2. Representación esquemática de las amplificaciones de VVZ en muestras sucesivas de humor acuoso. Las tres muestras fueron extraídas en el equipo MagNa Pure Compact (Roche) utilizando MagNA Pure Compact Nucleic Acid Isolation Kit I para RNA/DNA y amplificadas ocupando el kit de Fast Track Diagnostics N9 (Siemens) que incluye el patógeno de interés. Las muestras fueron corridas en conjunto para poder comparar su respectivo umbral de ciclo, que corresponde a aquel punto donde la curva de amplificación se separa de la curva de referencia. A menor carga viral la amplificación es más tardía y el umbral de ciclo se desplaza a la derecha (flecha negra) fenómeno que es evidente con la tercera muestra (M3). Las muestras M1 a M3 fueron tomadas con una distancia de una semana cada una. Los valores respectivos fueron 27,9; 28,54 y 32,3. El control positivo se denota $\mathrm{C}+$ y el control negativo como C-. El umbral de ciclo no permite estimar la carga viral real, proceso que requiere curvas de calibración con una cantidad de material genético conocida, sin embargo, permite estimar semi-cuantitativamente estos cambios. RFU: Relative Fluorescence Units. reacción inflamatoria, con una mejoría leve de la agudeza visual $(\mathrm{AV}$; hasta 0,4$)$ lo que permitió una reducción de las dosis de prednisona en forma progresiva desde 40 a $10 \mathrm{mg} /$ día. La evaluación con tomografía de coherencia óptica reveló un engrosamiento macular y de la capa de fibras nerviosas medidas en el OI. El cuadro inflamatorio ocular disminuyó y la AGF demostró una persistencia de la isquemia retinal periférica (Figuras 3A, 3B y 3 C). El paciente reconsultó siete meses después de haber terminado el período programado de terapia con valaciclovir acusando una disminución de la $\mathrm{AV}$ progresiva de 10 días en el OI ( $\mathrm{AV}$ 0,2 OI), con miodesopsias pero sin dolor ocular o fotopsias. La BMC mostró precipitados retrocorneales gruesos y finos, sin fenómeno de Tyndall. El FO evidenció un humor vítreo denso y la ecografía ocular fue compatible con DR en el OI. Nunca hubo compromiso del ojo contralateral. Fue tratado con vitrectomía y aplicación de láser con gas, permitiendo controlar el DR pero con una AV $<0,05$ (capaz de contar dedos).

\section{Discusión}

La NRA corresponde a un cuadro de panuveitis provocada por diferentes integrantes de virus de la familia herpes como VHS- 1, VHS-2, VVZ o CMV ${ }^{1-3}$. Habitualmente ocurre por una reactivación viral y se manifiesta con dolor ocular, visión borrosa, percepción de cuerpos flotantes (miodesopsias) y pérdida periférica del campo 


\section{Caso Clínico}

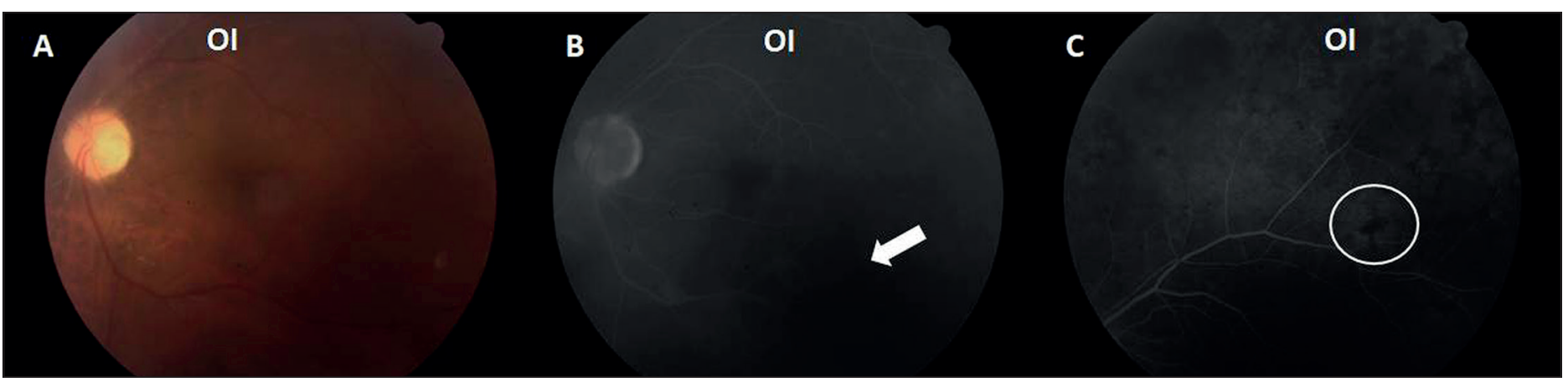

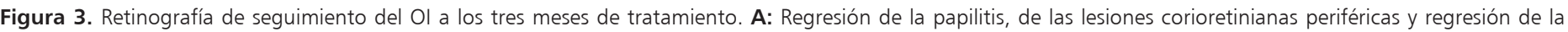

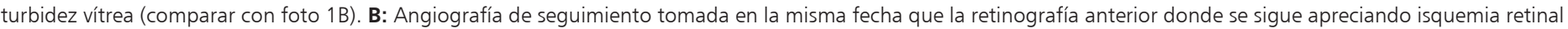

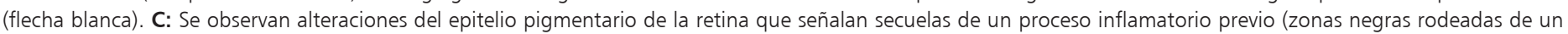
halo de fluoresceína; círculo blanco).

Tabla 1. Resumen de los aspectos diagnósticos y terapéuticos ante eventos de necrosis retiniana aguda

La NRA es una posible causa de panuveitis y está asociada mayoritariamente a reactivaciones de infecciones por VHS o VVZ. Es un evento de baja frecuencia pero asociada a un mal pronóstico visual a corto o mediano plazo

Se debe intentar el diagnóstico etiológico mediante RPC de una muestra de fluido ocular. La amplificación debe estar dirigida al menos a VHS 1-2 y VVZ

Descartar otras causas de panuveitis por anamnesis, examen físico y estudios complementarios, como por ejemplo: serología IgG/lgM toxoplasmosis, sífilis, tuberculosis (PPD o pruebas de liberación de interferon gamma, radiografía o tomografía computarizada de tórax), arañazo de gato (IgG Bartonella henselae), endocarditis infecciosa y enfermedades autoinmunes.

Evaluar posible inmunosupresión (VIH, linfopenia, antecedentes de trasplante de órganos o terapia corticoesteroidal)

En caso de sospecha, incluso sin confirmación etiológica, iniciar terapia con aciclovir intravenoso a dosis de $10 \mathrm{mg} / \mathrm{kg} / \mathrm{dosis}$ o $500 \mathrm{mg} / \mathrm{m}^{2}$ por dosis por 3 veces al día. Alternativamente (si está disponible), terapia oral con valaciclovir $1.500 \mathrm{mg}$ cada $8 \mathrm{~h}$. Esta última estrategia permite el alta precoz o terapia ambulatoria

Ajustar la dosis de aciclovir o valaciclovir según función renal

La duración recomendada del tratamiento es de tres meses para disminuir las recaídas, recurrencias o compromiso contralateral

La aplicación de la terapia intravítrea puede ser considerada como terapia coadyuvante, podría mejorar el pronóstico visual y durante el procedimiento permitiría obtener muestras para hacer un seguimiento indirecto de la carga viral en el tiempo mediante RPC

Los corticoesteroides sistémicos pueden ser considerados en procesos inflamatorios intensos

La oportunidad del tratamiento mejora el pronóstico, pero cerca de la mitad de los casos tendrán un compromiso visual grave a corto o mediano plazo

visual $^{1,6}$. El cuadro clínico es similar, independiente de su etiología y afecta tanto a pacientes inmunocomprometidos o inmunocompetentes ${ }^{1,6}$. Las causas predominantes corresponden al VHS y VVZ y su frecuencia relativa no depende de la edad ${ }^{6}$. En nuestro paciente otras causas de panuveitis fueron razonablemente descartadas por la historia clínica y exámenes complementarios tales como toxoplasmosis, sífilis, tuberculosis, enfermedad por arañazo de gato, toxocariasis o enfermedades autoinmunes.

Este caso permite revisar varios aspectos de la NRA relacionados a la etapa diagnóstica y el rol de la biología molecular, el manejo antiviral local y sistémico con sus respectivas consideraciones farmacocinéticas, la prevención del compromiso ocular contralateral y el pronóstico visual a largo plazo.

El diagnóstico precoz mediante RPC de una muestra de humor acuoso o vítreo es fundamental para confirmar el diagnóstico, identificar el agente involucrado y establecer el manejo y pronóstico. La sensibilidad de la RPC es elevada acercándose al 90-100\% ${ }^{1,3-5,7}$. La disponibilidad de técnicas cuantitativas ha permitido definir que una alta carga viral parece explicar una menor AV inicial y se asocia además a una mayor tasa de desprendimiento de retina ${ }^{4}$. 
Lo infrecuente de estos cuadros ha impedido efectuar evaluaciones comparativas sobre las mejores estrategias terapéuticas. Históricamente los cuadros de NRA por VVZ o VHS han sido tratados con aciclovir con una fase inicial por vía intravenosa usando dosis de 15 a $30 \mathrm{mg}$ / $\mathrm{kg} /$ día o $1.500 \mathrm{mg} / \mathrm{m}^{2} /$ día en tres dosis, por al menos una semana seguido de un período de mantención de uno a tres meses ${ }^{3,6-8}$. El objetivo de la fase inicial es detener el cuadro inflamatorio y preservar la visión; y el de la fase de mantención es prevenir la recaída y el compromiso del ojo contralateral. La importancia de un tratamiento prolongado proviene de un estudio observacional que reveló una reducción en la tasa de compromiso contralateral en $57 \%$ al prolongar el tratamiento por al menos cuatro semanas luego de la inducción ${ }^{8}$.

Observaciones y estimaciones farmacocinéticas han establecido que una dosis de $1 \mathrm{~g}$ cada $6 \mathrm{~h}$ de valaciclovir, la prodroga de aciclovir permite concentraciones plasmáticas similares a las obtenidas con dosis de aciclovir intravenoso de $5 \mathrm{mg} / \mathrm{kg} / 8 \mathrm{~h}$ y que dosis de valaciclovir de 2 g cada $6 \mathrm{~h}$ logran equivalencias farmacocinéticas con dosis por vía intravenosa de $10 \mathrm{mg} / \mathrm{kg} / 8 \mathrm{~h}^{9}$. Las concentraciones de aciclovir en el humor vítreo en ausencia de inflamación ocular alcanzan un $24 \%$ de sus concentraciones plasmáticas y dosis de $3 \mathrm{~g}$ al día de valaciclovir logran una concentración local de aciclovir de $1 \mu \mathrm{g} / \mathrm{ml}^{10}$. Esta cifra se estima suficiente para inhibir el crecimiento tisular de la mayor parte de las cepas de VHS-1, VHS-2 o VVZ $3,10,11$. En la práctica clínica no se dispone de información sobre las concentraciones requeridas de aciclovir para inhibir la cepa involucrada en cada caso, por lo que las dosis escogidas reflejan la dispersión existente en la literatura especializada, la experiencia local y el deseo de minimizar el margen de error. En forma más reciente, Liu y cols. utilizando la información disponible y modelos matemáticos, estimaron que la dosis óptima necesaria para un episodio de NRA por VVZ (y por extensión para VHS-1 y 2) corresponde a $1.500 \mathrm{mg}$ de valaciclovir cada $8 \mathrm{~h}$, superior a la dosis estándar de $1.000 \mathrm{mg}$ cada $8 \mathrm{~h}$ para infecciones cutáneas $^{11}$. Esta posología es equivalente a los $30 \mathrm{mg} /$ $\mathrm{kg} /$ día de aciclovir intravenoso y su gran ventaja es que permite un tratamiento oral desde el comienzo, facilitando el manejo ambulatorio o una corta estadía hospitalaria? No es recomendable actualmente aplicar terapias con aciclovir oral debido a su menor biodisponibilidad con pobres concentraciones plasmáticas en relación a las logradas con valaciclovir. El tratamiento coadyuvante con ganciclovir (2-5 mg/0,1 ml) o foscarnet $(2,4 \mathrm{mg} / 0,1 \mathrm{ml})$ intravítreo, como ocurrió en nuestro paciente, se aplica hasta en un tercio de los $\operatorname{casos}^{7} \mathrm{y}$ en estudios observacionales se ha asociado a una mejor recuperación de la AV y a una menor tasa de $\mathrm{DR}^{3,12}$. La larga estadía hospitalaria en nuestro paciente fue explicada por la aplicación de esta terapia y por las gestiones necesarias para conseguir valaciclovir, fármaco no disponible en el arsenal del hospital. Los corticoesteroides sistémicos como en nuestro caso, pueden ser considerados en procesos inflamatorios intensos que contribuyen a la pérdida de visión y que involucran casos de vitritis intensa, desprendimiento seroso de retina con compromiso macular, retinitis o vasculitis oclusiva que afecta el nervio óptico o mácula² .

En relación a la biología molecular, en este caso la RPC no sólo se utilizó en la fase diagnóstica sino que también para monitorizar la respuesta al tratamiento. Los datos indican que el aumento del umbral de ciclo en el seguimiento del paciente, sugiere una posible disminución en la cantidad de copias de material genético detectadas por la RPC del VVZ, lo que ocurrió sólo después de la segunda semana de tratamiento combinado, en paralelo al control de la inflamación y recuperación parcial de la AV. No tenemos antecedentes de otros casos de NRA donde se haya efectuado un seguimiento longitudinal semi-cuantitativo a través del umbral de ciclo el que no es equivalente a la carga viral ${ }^{13}$. Desafortunadamente, el control microbiológico no impidió a mediano plazo el DR.

El pronóstico de la NRA es sombrío ya que cerca de la mitad de los casos presentarán una pérdida de visión grave en los meses posteriores. Los factores asociados a este desenlace están relacionados a la intensidad de la pérdida de la $\mathrm{AV}$, a la demora en el inicio del tratamiento, al desarrollo de un DR, a una alta carga viral inicial y a $\mathrm{VVZ}^{4,7,12}$.

\section{Referencias bibliográficas}

1.- Torres J P, López J P. Necrosis retinal aguda: Una entidad de adultos en pediatría. Rev Chilena Infectol 2007; 24: 327-30. doi: $10.4067 / \mathrm{S} 0716-10182007000400012$.

2.- $\quad$ Wong R W, Jumper J M, McDonald H R, Johnson R N, Fu A, Lujan B J, et al. Emerging concepts in the management of acute retinal necrosis. Br J Ophthalmol 2013; 97: 545-52. doi: 10.1136/bjophthalmol-2012-301983.
3.- Schoenberger S D, Kim S J, Thorne J E, Mruthyunjaya P, Yeh S, Bakri S J, et al. Diagnosis and treatment of acute retinal necrosis: A report by the American Academy of Ophthalmology. Ophthalmology 2017; 124: 382-92. doi: 10.1016/j.ophtha.2016.11.007.

4.- Calvo C M, Khan M A, Mehta S, Garg S J, Dunn J P. Correlation of clinical outcomes with quantitative polymerase chain reaction DNA copy number in patients with acute retinal necrosis. Ocul
Immunol Inflamm 2017; 25: 246-52. doi: 10.3109/09273948.2015.1115081.

5.- Butler N J, Moradi A, Salek S S, Burkholder B M, Leung T G, Dunn J P, et al. Acute retinal necrosis: presenting characteristics and clinical outcomes in a cohort of polymerase chain reaction-positive patients. Am J Ophthalmol 2017; 179: 179-89. doi: 10.1016/j. ajo.2017.05.006.

6.- Ittner E, Bahkhri R, Newman T. Necrotising herpetic retinopathies: a review and progressive 


\section{Caso Clínico}

outer retinal necrosis case report. Clin Exp Optom 2016; 99: 24-9. doi: 10.1111/cxo.12284.

7.- Baltinas J, Lightman S, Tomkins-Netzer O. Comparing treatment of acute retinal necrosis with either oral valaciclovir or intravenous aciclovir. Am J Ophthalmol 2018; 188: 173-80. 10.1016/j.ajo.2018.02.001

8.- Palay D A, Sternberg P Jr, Davis J, Lewis H, Holland G N, Mieler WF, et al. Decrease in the risk of bilateral acute retinal necrosis by acyclovir therapy. Am J Ophthalmol 1991; 112: 250-5. doi: 10.1016/s0002-9394(14)76725-x.

9.- Weller S, Blum M R, Doucette M, Burnette
T, Cederberg D M, de Miranda P, et al. Pharmacokinetics of the acyclovir pro-drug valaciclovir after escalating single- and multiple-dose administration to normal volunteers. Clin Pharmacol Ther 1993; 54: 595605. doi: 10.1038/clpt.1993.196.

10.- Huynh T H, Johnson M W, Comer G M, Fish D $\mathrm{N}$. Vitreous penetration of orally administered valacyclovir. Am J Ophthalmol 2008; 145: 6826. doi: 10.1016/j.ajo.2007.11.016.

11.- Liu T, Jain A, Fung M, Vinnard C, Ivaturi V. Valacyclovir as initial treatment for acute retinal necrosis: a pharmacokinetic modeling and simulation study. Curr Eye Res 2017; 42: 1035-8. doi: 10.1080/02713683.2016.1231323. 12.- Wong R, Pavesio C E, Laidlaw D A, Williamson T H, Graham E M, Stanford M R. Acute retinal necrosis: the effects of intravitreal foscarnet and virus type on outcome. Ophthalmology 2010; 117: 556-60. doi: 10.1016/j.ophtha.2009.08.003.

13.- Aquino-Jarquin G. The raw cycle threshold values from reverse-transcription polymerase chain reaction detection are not viral load quantitation units. Clin Infect Dis 2021; 72 : 1489-90. doi: 10.1093/cid/ciaa830. 